\title{
Avaliação dos resultados tardios da operaçãoo de derivação cavo-pulmonar bidirecional, no tratamento paliativo de cardiopatias congênitas com câmara ventricular única
}

Miguel Angel MALUF* ${ }^{\star}$, José carlos S. ANDRADE* ${ }^{*}$ Roberto CATANI*, Antônio C. CARVALHO* Nilce NEGRINI, Ênio BUFFOLO*

MALUF, M. A.; ANDRADE, J. C. S.; CATANI, R.; CARVALHO, A. C.; NEGRINI, N.; BUFFOLO, E. - Avaliaçāo dos resultados tardios da operação de derivação cavo-pulmonar bidirecional, no tratamento paliativo de cardiopatias congênitas com câmara ventricular única. Rev. Bras. Cir. Cardiovasc., 9 (2): 95-103, 1994.

RESUMO: No periodo de março de 1990 a janeiro de 1994, 17 pacientes com idades de 1 a 13 anos (média: 7 anos), portadores de cardiopatias congênitas com câmara ventricular única funcionante, foram submetidos a operaçāo de derivaçāo cavo-pulmonar bidirecional. Nove pacientes tinham atresia tricúspide (AT), 7 do tipo lb, 1 tipo lc e 1 tipo llb. Cinco pacientes tinham dupla via de entrada ventricular tipo ventrículo direito (DVEVD); 2 com comunicação interventricular (CIV) múltipla+hipoplasia do VD; 1 com atresia pulmonar com septo interventricular integro (APc/SIVI). Nove $(52,9 \%)$ pacientes tinham operaçōes paliativas prévias. A operação foi realizada com auxilio da circulação extracorpórea (CEC) em $12(70,5 \%)$ casos e mediante derivação do fluxo sangūíneo da veia cava superior (VCS) para o átrio direito (AD) em $5(29,5 \%)$ casos. Em todos os casos a VCS foi anastomosada à artéria pulmonar direita (APD), interrompendo o fluxo sangūineo para o pulmão, fechando a valva pulmonar e ligando a derivação de Blalock-Taussig pérvio. Houve 3(17,6\%) óbitos no pós-operatório imediato (POI) e $2(14,2 \%)$ no pós-operatório tardio (POT). Doze $(70,5 \%)$ pacientes estão em acompanhamento clínico, com um tempo de evolução de 2 a 46 meses. Um paciente foi submetido ao $2^{\circ}$ tempo da operaçāo, tunelizando a veia cava inferior $(\mathrm{VCl})$ para a APD, com sucesso. A avaliaçāo do fluxo da derivação cavo-pulmonar bidirecional está sendo realizada pela ecodopplercardiografia e ressonância nuclear magnética e a perfusāo pulmonar mediante cintilografia radioisotópica. A indicação do $2^{\circ}$ tempo da operaçāo obedece à própria evoluçāo clínica e avaliaçāo da saturação arterial durante a cicloergometria. A derivação cavo-pulmonar bidirecional permite uma adaptaçăo progressiva do fluxo venoso para o pulmão, diminuindo a sobrecarga de volume do ventriculo, preparando o paciente para a derivaçāo venosa total.

DESCRITORES: derivação cavo-pulmonar, cirurgia

\section{INTRODUÇÃO}

A correção cirúrgica de cardiopatias congênitas que apresentam câmara ventricular única funcionante implica no emprego de técnicas paliativas, que orientam o fluxo venoso sistêmico para o pulmão, desativando o mecanismo pulsátil do ventrículo pulmonar (hipoplásico e impróprio).

GLENN, em $1958^{7}$, demonstrou a possibilidade de realizar uma derivação cavo-pulmonar, anastomosando a veia cava superior (VCS) com a artéria

Trabalho realizado na Disciplina de Cirurgia Cardiovascular da Escola Paulista de Medicina. São Paulo, SP, Brasil.

Apresentado ao $21^{2}$ Congresso Nacional de Cirurgia Cardiaca. Porto Alegre, RS, 24 a 26 de março, 1994.

- Do Departamento de Cirurgia da Escola Paulista de Medicina.

Endereço para correspondência: Miguel A. Maluf; Escola Paulista de Medicina, Departamento de Cirurgia. Rua Napoleão de Barros, 715. CEP 04024002 Vila Clementino. Fax:(011) 571-2719. São Paulo, SP, Brasil. 
MALUF, M. A.; ANDRADE, J. C. S.; CATANI, R.; CARVALHO, A. C.; NEGRINI, N.; BUFFOLO, E. - Avaliaçāo dos resultados tardios da operação de derivaçāo cavo-pulmonar bidirecional, no tratamento paliativo de cardiopatias congênitas com câmara ventricular única. Rev. Bras. Cir. Cardiovasc., 9 (2): 95-103, 1994.

pulmonar direita (APD) com técnica término-terminal e interrompendo a continuidade das artérias pulmonares.

HALLER et alii 8 provaram experimentalmente a possibilidade de realizar uma anastomose entre a VCS e APD, mantendo fluxo pulmonar bidirecional.

A aplicação clínica deste procedimento foi realizada por ABRAMS ${ }^{1}$, não sendo esta alternativa adequadamente explorada até 1984 , quando KAWASHIMA et alii ${ }^{11}$ mostraram os resultados da anastomose cavo-pulmonar em cardiopatias complexas, com drenagem da veia cava inferior $(\mathrm{VCl})$ através da veia ázigos ou hemiazigos.

Após a operação de FONTAN \& BAUDET 6 , num paciente portador de atresia tricúspide, têm sido incorporadas várias modificações no conceito original $9,10,12,18,19$. Este procedimento de derivação total do retorno venoso sistêmico para o pulmão requer uma cuidadosa avaliação das condições anatomofuncionais do coração e árvore pulmonar, critérios estes que foram descritos por CHOUSSAT et alii ${ }^{2}$.

Apesar da análise detalhada dos critérios de indicação e apurada técnica cirúrgica, os resultados imediatos da derivação cavo-pulmonar total acarretam mortalidade de $20 \%$ a $30 \%{ }^{19}$.

Com o intuito de reduzir os indices de mortalidade, alguns grupos preferiram realizar a correção em estágios, baseados em operaçōes que haviam demonstrado boa evolução dos pacientes a longo prazo.

A vantagem de uma derivação cavo-pulmonar bidirecional é o aumento efetivo do fluxo venoso sistêmico para o pulmão, com diminuição da sobrecarga de volume do ventriculo, sem causar doença pulmonar obstrutiva. Ao contrário, o fluxo pulmonar é suprido pela drenagem da VCS, após ligadura da derivação de Blalock-Taussig ou tronco pulmonar (TP).

TABELA 1

PACIENTES PORTADORES DE CARDIOPATIAS CONGÊNITAS CIANÓTICAS COMPLEXAS, SUBMETIDOS A OPERAÇÄO DE ANASTOMOSE CAVO-PULMONAR BIDIRECIONAL

\begin{tabular}{|c|c|c|c|c|c|c|c|c|c|c|}
\hline \multirow[t]{2}{*}{$N^{0}$} & \multirow[t]{2}{*}{ NOME } & \multirow[t]{2}{*}{$1 D$} & \multirow[t]{2}{*}{ DIAGNÓSTICO } & \multirow[t]{2}{*}{ OP. PRÉVIA } & \multirow[t]{2}{*}{ TIPO OP. } & \multirow[t]{2}{*}{ CEC } & \multirow[t]{2}{*}{ DATAOP. } & \multicolumn{3}{|c|}{ OBITOS } \\
\hline & & & & & & & & $\mathrm{POI}$ & & CAUSAS \\
\hline 1 & J.A.S. & 9 & A.T.+T.G.A.+E.P. & B-T.-d. & A.cavo-pul.bid. & $\mathrm{s}$ & $29 / 03 / 90$ & - & - & - \\
\hline 2 & C.L.O. & 1 & Hipo.VD+CIV+EP & - & A.cavo-pul.bid. & $\mathrm{s}$ & $26 / 07 / 90$ & - & - & - \\
\hline 3 & R.G. & 5 & DVEVD+V.S-I+EP & - & A.cavo-pul.bid. & $\mathrm{s}$ & $15 / 02 / 91$ & - & - & - \\
\hline 4 & A.C.T. & 11 & A.T. $-\mathrm{Ib}$ & B.T.-e. & A.cavo-pul.bid. & $\mathrm{N}$ & $16 / 07 / 91$ & - & - & - \\
\hline 5 & D.X.S. & 3 & A.T.-I.C. & Band.TP & A.cavo-pul.bid & $\mathrm{s}$ & $23 / 10 / 91$ & - & $\mathrm{s}$ & $\begin{array}{l}\text { Morte } \\
\text { súbita }\end{array}$ \\
\hline 6 & G.P.S. & 11 & A.T.+DSA-V+IM & B.T.-e & A.cavo-pul.bid. & $\mathrm{N}$ & $12 / 02 / 92$ & - & - & - \\
\hline 7 & F.M.O. & 12 & A.T. $-1 \mathrm{~b}$ & - & A.cavo-pul.bid. & $\mathrm{S}$ & $14 / 04 / 92$ & $\mathrm{~S}$ & - & S.B.D. \\
\hline 8 & L.A.B. & 5 & A.T. $-1 \mathrm{~b}$ & - & A.cavo-pul.bid. & $\mathrm{S}$ & $14 / 05 / 92$ & - & $\cdot$ & $\cdot$ \\
\hline 9 & D.O.B. & 5 & Hipo.VD+CIV Mult. & Band. TP & A.cavo-pul.bid. & $\mathrm{S}$ & $29 / 06 / 92$ & $\mathrm{~S}$ & - & S.B.D. \\
\hline 10 & S.G.P. & 5 & A.T. $-1 \mathrm{~b}$ & B.T.-d.+e. & $\begin{array}{l}\text { A.cavo-pul.bid. } \\
\text { +A.Ap e. }\end{array}$ & s & $17 / 08 / 92$ & - & - & - \\
\hline 11 & F.S.L. & 11 & A.P. $c / S I V I$ & $\begin{array}{l}\text { B.T.d.+e.t } \\
\text { A.VSVD }\end{array}$ & A.cavo-pul.bid. & $\mathrm{s}$ & $19 / 01 / 93$ & - & - & - \\
\hline 12 & H.R.L. & 8 & DVEVD+TGA & Band.TP & $\begin{array}{l}\text { A.cavo- } \\
\text { pul.bid.+A.CIA }\end{array}$ & $\mathrm{S}$ & $10 / 02 / 93$ & - & $\mathrm{S}$ & $\begin{array}{l}\text { Tromb. } \\
\text { ven. }\end{array}$ \\
\hline 13 & M.V.O. & 3 & $\begin{array}{l}\text { DVEVD+At.Mitr+D } \\
\text { APVP+EP }\end{array}$ & - & $\begin{array}{l}\text { A.cavo- } \\
\text { pul.bid.+A.CIA }\end{array}$ & $\mathrm{S}$ & $12 / 02 / 93$ & $S$ & - & S.B.D. \\
\hline 14 & D.S.A. & 3 & DVEVD+TGA+EP & $\cdot$ & $\begin{array}{l}\text { A.cavopul.bid } \\
\text { +A.AP e. }\end{array}$ & s & $28 / 06 / 93$ & - & - & - \\
\hline 15 & C.B. & 13 & DVEVD+EP & - & A.cavo-pul.bid. & $\mathrm{N}$ & $06 / 07 / 93$ & - & - & - \\
\hline 16 & R.M.S. & 6 & A.T. $-1 \mathrm{~b}$ & B.T.-e. & A.cavo-pul.bid. & $N$ & $17 / 01 / 94$ & - & - & - \\
\hline 17 & T.B.N. & 6 & A.T. $-1 \mathrm{~b}$ & - & A.cavo-pul.bid. & $\mathrm{N}$ & $31 / 01 / 94$ & - & - & - \\
\hline
\end{tabular}

AT: atresia tricúspide; TGA: Transposição das grandes artérias; EP: Estenose Pulmonar; Hipo-VD: Hipoplasia do Ventriculo Direito CIV: Comunicação Interventricular; DVE-VD: Dupla Via de Entrada do Ventrículo Direito; IM:Insuficiência Mitral; AP c/ SIVI: Atresia Pulmonar com Septo Interventricular Íntacto; DAPVP: Drenagem Anômala Parcial de Veias Pulmonares; V.S.-I: ventrículo Sup.Inferior; BT: Blalock-Taussig; Band. TP: Bandagem do Tronco Pulmonar; A.: Ampliação; ClA: Comunicação Interatrial; Mult: Múltipla; S.B.D.: Síndrome de Baixo Débito; DSA-V: Defeito Septal Átrioventricular. 
MALUF, M. A.; ANDRADE, J. C. S.; CATANI, R.; CARVALHO, A. C.; NEGRINI, N.; BUFFOLO, E. - Avaliação dos resultados tardios da operação de derivação cavo-pulmonar bidirecional, no tratamento paliativo de cardiopatias congênitas com câmara ventricular única. Rev. Bras. Cir. Cardiovasc., 9 (2): 95-103, 1994.

Este trabalho tem como objetivo mostrar os resultados da avaliação tardia de pacientes portadores de cardiopatias congênitas complexas submetidos a derivação cavo-pulmonar bidirecional, sem possibilidades de correção biventricular.

\section{CASUISTICA E MÉTODOS}

De março de 1990 a janeiro de 1994,17 pacientes com idades de 1 a 13 anos (média: 7 anos), portadores de cardiopatia congênita cianótica complexa, com câmara ventricular única funcionante, foram submetidos a operação de derivação cavopulmonar bidirecional.

Nove $(53 \%)$ pacientes tinham atresia tricúspide (AT): 7 casos tipo lb ( 1 caso com defeito septal atrioventricular [DSA-V] + insuficiência mitral), 1 tipo Ic e 1 tipo IIb; $5(29,5 \%)$ casos tinham dupla via de entrada ventricular tipo ventrículo direito (DVEVD): $3 \mathrm{com}$ estenose pulmonar (EP), $1 \mathrm{com}$ hiperfluxo pulmonar e 1 com EP+atresia mitral; $2(11,7 \%)$ casos com comunicação interventricular (CIV) múltipla e hipoplasia do VD e $1(5,8 \%)$ caso atresia pulmonar com septo interventricular integro (APc/SIVI).

Nove $(52,9 \%)$ pacientes tinham operações paliativas prévias: tipo Blalock-Taussig (B-T) unilateral em 4 casos; B-T bilateral em 2 casos com ampliação da via de saída do ventrículo direito (VSVD) em 1 caso e bandagem do tronco pulmonar (TP) em 3 casos (Tabela 1).

A indicação da derivação cavo-pulmonar foi baseada em critérios clínicos e anatômicos em pacientes maiores de 1 ano. Tratava-se de pacientes sintomáticos, onde apenas um ventrículo tinha conecção atrioventricular, sendo este de tamanho e desempenho suficiente para manter os débitos sistêmico e pulmonar. Em alguns casos, a escolha desta técnica foi devido a falta de opções, após ter esgotado os recursos de outras operações paliativas.
A operação foi realizada após adequada monitorização hemodinâmica, em especial com cateter posicionado na VCS.

A abordagem foi mediante toracotomia mediana, procedendo-se ao preparo do campo operatório, com dissecção da veia cava superior, veia inominada, artéria pulmonar direita e tronco pulmonar.

As derivações sistêmico-pulmonares tipo B-T foram cuidadosamente dissecadas e cadarçadas, para posterior ligadura.

Em $12(70,5 \%)$ casos o procedimento foi realizado com auxilio da circulação extracorpórea (CEC), em normotermia sem pinçamento da aorta. A drenagem venosa foi realizada mediante canulação da VCS na sua porção mais alta ou da veia inominada e do AD. O retorno do fluxo arterial foi feito usandose cânula posicionada na Ao ascendente.

Nos $5(29,5 \%)$ casos restantes, foi dispensado o uso da CEC, estabelecendo-se um curto-circuito temporário entre a VCS e $A D$, com cânulas de diâmetro adequado e com controle da pressão venosa central (PVC).

Para a realização da derivação cavo-pulmonar, foi seccionada a VCS na junção átrio-cava e suturado o coto proximal; aberta a APD no seu eixo longitudinal, realizando a anastomose entre essas duas estruturas com fio absorvível (Polydioxanona $=$ PDS) 6-0. A seguir, foi interrompido o fluxo sangüíneo para o pulmão, mediante ligadura do TP ou sutura da valva pulmonar (Figura 1).

Em 2 pacientes (casos 10 e 14) foi necessário a ampliação da artéria pulmonar esquerda (APE), devido a estenose localizada, sendo usado retalho de pericárdio. Em outros 2 (casos 12 e 13), foi ampliada a comunicação interatrial (CIA), com abordagem direta através de atriotomia mínima e com pinçamento temporário da aorta.

Os tempos de CEC variaram entre 20 e 65

TABELA 2

PACIENTES QUE EVOLUIRAM PARA ÓBITO NO PÓS-OPERATÓRIO IMEDIATO

\begin{tabular}{|c|c|c|c|c|c|c|c|c|c|}
\hline $\begin{array}{c}N^{Q} \\
\text { ORDEM }\end{array}$ & NOME & $\begin{array}{l}\text { IDADE } \\
\text { (ANOS) }\end{array}$ & DIAGNÓSTICO & PAM & $\begin{array}{c}P A D \\
(M M . H G)\end{array}$ & $P A E$ & $\begin{array}{c}\text { SAT } 02 \\
(\%)\end{array}$ & INTER. UTI & CONDUTA \\
\hline 7 & F.M.O. & 12 & At.-I B & $<50$ & $>17$ & $<12$ & $<80$ & S.B.D. & Takedown \\
\hline 9 & D.O.B. & 5 & $\begin{array}{l}\mathrm{H} . \mathrm{VD}+\mathrm{CIV} \\
\text { múlt. }\end{array}$ & $<50$ & $>17$ & $<12$ & $<80$ & $\begin{array}{l}\text { S.B.D.+ } \\
\text { PCR }\end{array}$ & Takedown \\
\hline 13 & M.V.O. & 3 & $\begin{array}{c}\text { DVEVD+At. } \\
\text { M.+DAPVP+ } \\
\text { EP }\end{array}$ & $<50$ & $>17$ & $<12$ & $<80$ & $\begin{array}{c}\text { S.B.D.+ } \\
\text { PCR }\end{array}$ & Reab. Tórax \\
\hline
\end{tabular}

PAM: Pressão arterial média; PAD: Pressão átrio direito; PAE: Pressão átrio esquerdo; SVD: Síndrome de Baixo Débito; PCR: Parada cardiorespiratória. 
MALUF, M. A.; ANDRADE. J. C. S.; CATANI, R.; CARVALHO, A. C.: NEGRINI, N.; BUFFOLO. E. - Avaliaçāo dos resultados tardios da operação de derivação cavo-pulmonar bidirecional, no tratamento paliativo de cardiopatias congênitas com câmara ventricular única. Rev. Bras. Cir. Cardiovasc., 9 (2): 95-103, 1994.

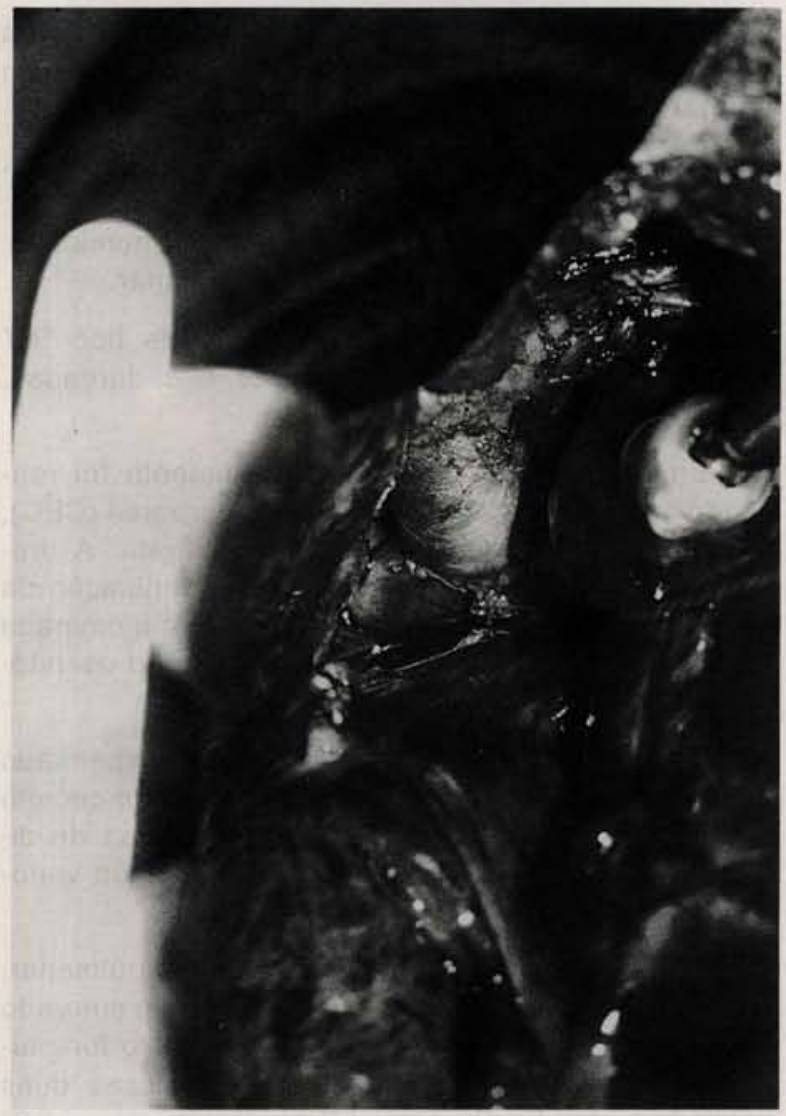

Fig. 1 - Fotografia do campo operatório. Anastomose da veia cava superior (VCS) com a artéria pulmonar direita (APD).

minutos. A saída de CEC foi em forma gradual, após adequada revisão da hemostasia da anastomose e avaliação do desempenho hemodinâmico e oximétrico do paciente.

A monitorizaçāo da PVC foi contínua, até adequada reposição volêmica. Na maioria das vezes, foi necessário o uso de drogas vasodilatadoras (nitroprussiato de sódio).
A saturação arterial de $\mathrm{O}_{2}$ oscilou entre $70 \%$ e $90 \%$. Durante o pós-operatório imediato (POI), alguns cuidados especiais foram tomados, no intuito de facilitar a melhor adaptaçāo da circulaçāo pulmonar, evitando: estase venosa, queda da perfusão pulmonar, insaturação arterial e baixo débito cardiaco.

Os pacientes foram mantidos em posição semisentada, sem sedação, intubados com ventilação mandatória intermitente (VMI) e pressão expiratória final positiva (PEEP) de zero.

A reposição volêmica foi feita sempre com colóides (albumina, plasma) ou sangue. Foram usadas drogas inotrópicas (dobutamina) e vasodilatadores (nitroprussiato de sódio) para melhorar as condições hemodinâmicas.

\section{RESULTADOS}

Os 14 pacientes nos quais os níveis de PVC estiveram abaixo de $17 \mathrm{mmHg}$ evoluíram bem. Outros 3 pacientes apresentaram estase jugular e elevação da PVC (18 a $20 \mathrm{~mm}$ ) no POI, seguido de quadro de baixo débito cardíaco, queda da saturação arterial $(<70 \%)$ e acidose metabólica de repetição; todos os 3 pacientes foram reoperados de imediato, sendo em 2 desfeita a anastomose (takedown) e em 1 foi feita reanimação cardiorespiratória, com tórax aberto. Nenhum paciente sobreviveu $(17,6 \%)$ (Tabela 2).

A evolução clínica foi satisfatória em 13 (76,4\%) pacientes, não sendo detectados derrames serosos na fase de internação hospitalar.

Um paciente (caso de $n^{2} 12$ ) apresentou trombose da veia jugular interna esquerda e inominada, no $7^{\circ}$ dia de $\mathrm{PO}$ e desenvolveu quilotórax à esquerda, sendo tratado com eparinização sistêmica e drenagem pleural.

Dois pacientes foram reoperados: 1 paciente

TABELA 3

PACIENTES EM ACOMPANHAMENTO CLINICO PÓS-OPERATÓRIO, SUBMETIDOS A REOPERAÇÃO, APÓS DERIVAÇÃO CAVO-PULMONARBIDIRECIONAL

\begin{tabular}{|c|c|c|c|c|c|c|c|c|c|}
\hline$N^{2}$ & NOME & $\begin{array}{l}\text { IDADE } \\
\text { (ANOS) }\end{array}$ & DIAGNÓSTICO & $\begin{array}{c}\text { DATA } \\
\left(1^{\text {a } O P)}\right.\end{array}$ & TIPO & EVOLUÇĀO & $\begin{array}{l}\text { DATA } \\
\text { REOP. }\end{array}$ & TIPO & EVOL. \\
\hline 6 & G.P.S. & 10 & $A T+D S A V+I M$ & $12 / 02 / 92$ & $\begin{array}{l}\text { A.cavo } \\
\text { pul.bid. }\end{array}$ & Ins. Mitral & $30 / 06 / 93$ & $\begin{array}{l}\text { Plastia Mitr } \\
\text { Troca } \\
\text { Mitr+Ampl.APE } \\
\text { + Lig. TP }\end{array}$ & Boa \\
\hline 12 & H.R.L. & 8 & DVEVD+TGA & $10 / 02 / 93$ & $\begin{array}{l}\text { A.cavo } \\
\text {-pul.bid }\end{array}$ & $\begin{array}{l}\text { Tromb. ven. } \\
\text { +Quilot. }\end{array}$ & $18 / 03 / 93$ & $\begin{array}{l}\text { Tromb.+ } \\
\text { Lig.TP }\end{array}$ & Óbito \\
\hline
\end{tabular}


MALUF, M. A.; ANDRADE, J. C. S.; CATANI, R.; CARVALHO, A. C.; NEGRINI, N.; BUFFOLO, E. - Avaliação dos resultados tardios da operação de derivação cavo-pulmonar bidirecional, no tratamento paliativo de cardiopatias congênitas com câmara ventricular única. Rev. Bras. Cir. Cardiovasc., 9 (2): 95-103, 1994.

TABELA 4

PACIENTES QUE EVOLUIRAM PARA ÓBITO NO POI

\begin{tabular}{ccccccc}
\hline NoORDEM & NOME & IDADE & DIAGNOSTICO & EVOLUÇÃOPO & \multicolumn{1}{c}{ B BITO } \\
\hline 5 & D.X.S & 4 & At.-I.C. & Morte Súbita & $12 \mathrm{~m}$ CAUSA & Desconhecida \\
12 & H.R.L. & 8 & DVEVD+TGA+Dex. & $\begin{array}{c}\text { Tromb. } \\
\text { venos.+Quilot. }\end{array}$ & $\begin{array}{c}41 d \\
\text { Baixo Débito } \\
\text { Cardiaco }\end{array}$ \\
\hline
\end{tabular}

(caso de $n \div 6$ ) apresentava insuficiência mitral após um ano, teve piora do grau funcional, sendo submetido a plastia seguida, de substituição valvar, com boa evolução. Outro paciente (caso de $n$ ㅇ 12) foi reoperado para a remoção de trombo venoso que comprometia a anastomose cavo-pulmonar no $36^{\circ}$ dia de $\mathrm{PO}$, evoluindo com baixo débito cardíaco (Tabela 3).

Houve dois $(14,2 \%)$ óbitos no POT: caso de $n^{\circ}$ 12 no $41^{\circ}$ dia de PO (após reoperação) e caso de $\mathrm{n} \div 5$, no $12^{\circ}$ mês de $\mathrm{PO}$ por morte súbita, não tendo sido feito exame necroscópico, (Tabela 4).

Doze $(70,5 \%)$ pacientes sobreviventes estāo em acompanhamento clínico com evolução de 2 a 46 meses, sem uso de drogas anticoagulantes ou antiagregantes plaquetários.

Um paciente (caso de $n^{\circ} 2$ ) foi submetido ao segundo tempo da operação (túnel VCI-APD), com sucesso.

Onze pacientes submetidos a ecodopplercardiografia seriada, mostraram bom fluxo através da derivação cavo-pulmonar e 2 apresentam fluxo dis- creto através da valva pulmonar. Três pacientes foram submetidos a estudo de ressonância nuclear magnética, detectando-se bom funcionamento da derivação (Figura 2, Tabela 5).

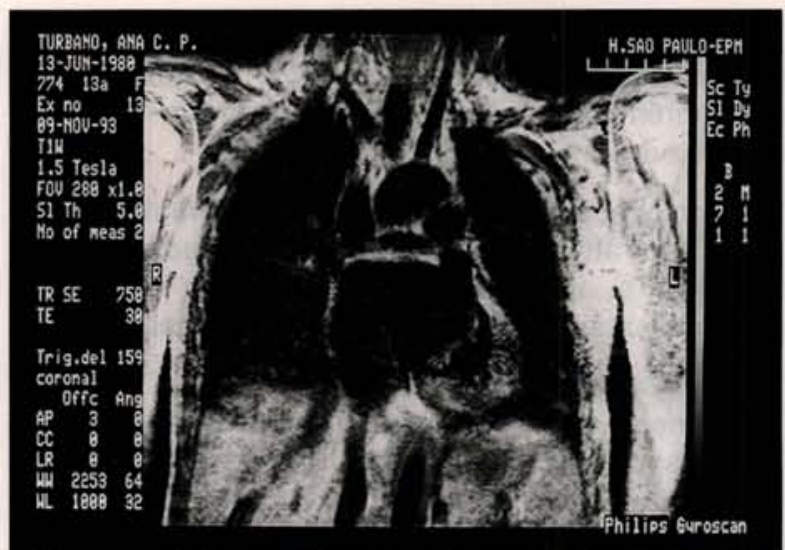

Fig. 2 - Ressonância nuclear magnética no pós-operatório. Observa-se fluxo sangüineo da veia cava superior (VCS) para artéria pulmonar direita (APD), sem obstáculos ao nivel da anastomose.

TABELA 5

PACIENTES EM ACOMPANHAMENTO CLINICO PÓS-OPERATORIO, SUBMETIDOS A AVALIAÇÃO DA DERIVAÇÃO CAVOPULMONARBIDIRECIONAL

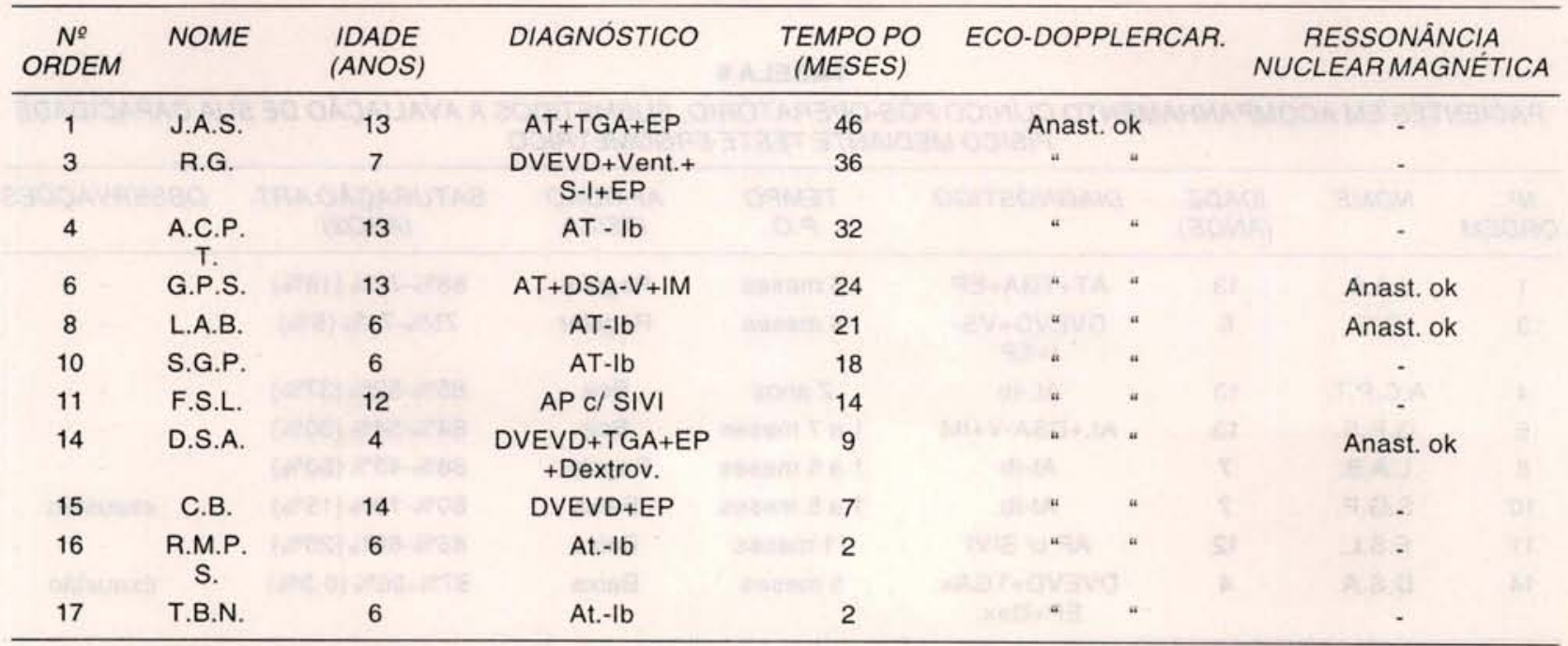


MALUF, M. A.; ANDRADE, J. C. S.; CATANI, R.; CARVALHO, A. C.; NEGRINI, N.; BUFFOLO, E. - Avaliação dos resultados tardios da operaçāo de derivação cavo-pulmonar bidirecional, no tratamento paliativo de cardiopatias congênitas com câmara ventricular única. Rev. Bras. Cir. Cardiovasc., 9 (2): 95-103, 1994.

Oito pacientes, maiores de 4 anos realizaram teste ergométrico com esteira e controle simultâneo da saturação arterial; em 2 a atividade física foi considerada boa, em 3 regular e em 3 casos, baixa; os valores da saturação de 02 (S02) variaram de $5 \%$ a $50 \%$ (média: $26,6 \%$ ). Dois pacientes (casos de $n \div 10$ e $n \div 14$ ) não concluiram o teste, devido a exaustão (Tabela 6).

\section{COMENTÁRIOS}

A operação tipo Fontan-Kreutzer (KREUTZER et alii ${ }^{14}$ ), foi proposta para a correção paliativa de cardiopatias congênitas complexas, onde nāo seria possível o aproveitamento de ambos os ventrículos.

Desta forma, conseguiu-se uma correção fisiológica, separando o fluxo sistêmico do fluxo pulmonar, porém provocando diferentes graus de hipertensão venosa sistêmica.

A elevação da PVC, acima de $20 \mathrm{mmHg}$, associou-se a alta mortalidade, e o procedimento de desfazer a operação (takedown) não conseguiu mudar, muitas vezes, a evolução para óbito $3,4,17$.

Por outro lado a persistência de uma hipertensão venosa crônica levaria a formação de derrames serosos ou provocaria uma congestāo hepática crônica 5 .

Para o sucesso da operação, é necessário uma cuidadosa seleção de pacientes e uma acurada intervenção, evitando qualquer tipo de obstrução ao fluxo venoso sistêmico e do leito vascular pulmonar.

Os critérios originais de CHOUSSAT et alii ${ }^{2}$ para a indicação cirúrgica foram ampliados, incluindo-se pacientes com anomalias complexas. Os parâmetros como: pressão da artéria pulmonar (PAP) menor que $20 \mathrm{mmHg}$ e resistência vascular pulmonar (RVP) menor que 2 unidades Wood têm sido respeitados.

No entanto, pacientes que evoluem temporariamente com hipertensão pulmonar (antes da bandagem pulmonar), ou apresentam uma importante circulação colateral, ou casos com atresia mitral e CIA pequena, ou presença de insuficiência da valva A-V esquerda ou estenose subaórtica, constituem um grupo de alto risco para a operação de derivação cavo-pulmonar total.

Uma opção para este grupo de pacientes é a realização da operação por estágios. No primeiro tempo, é desviado o fluxo da VCS para o pulmão, mediante anastomose cavo-pulmonar bidirecional.

Uma segunda opção para este grupo de pacientes é a realização da operação de Fontan deixando uma fenestração no túnel da $\mathrm{VCl}$, descrito por LAKS et alii ${ }^{15}$ e considerado como operação parcial de Fontan (LAKS et alii ${ }^{16}$ ).

A partir de 1990 , estabelecemos no nosso Serviço a realização da derivação cavo-pulmonar por estágios.

A anastomose cavo-pulmonar bidirecional permitiu manter a saturação arterial em torno de $85 \%$, com pressão baixa no $A D$ e bom débito cardiaco. A adaptação progressiva do leito vascular pulmonar de apenas $1 / 3$ da circulação venosa sistêmica é melhor tolerada na primeira fase do $\mathrm{PO}$, onde $\mathrm{O}$ vasoespasmo pulmonar é freqüente.

A presença de PVC baixa evitou a formação de derrames serosos.

A derivação cavo-pulmonar bidirecional tem sido usada por KOBAYASHI et alii ${ }^{19}$, beneficiando pacientes considerados não ideais para a operação de Fontan.

TABELA 6

PACIENTES EM ACOMPANHAMENTO CLINIICO PÓS-OPERATÓRIO, SUBMETIDOS A AVALIAÇĀO DE SUA CAPACIDADE FISICO MEDIANTE TESTE ERGOMÉTRICO

\begin{tabular}{|c|c|c|c|c|c|c|c|}
\hline $\begin{array}{l}N^{2} \\
\text { ORDEM }\end{array}$ & NOME & $\begin{array}{l}\text { IDADE } \\
\text { (ANOS) }\end{array}$ & DIAGNÓSTICO & $\begin{array}{l}\text { TEMPO } \\
\text { P.O. }\end{array}$ & $\begin{array}{l}\text { APTIDĀO } \\
\text { FÍSICA }\end{array}$ & $\begin{array}{c}\text { SATURAÇĀO ART. } \\
\text { (ASO2) }\end{array}$ & OBSERVAÇŌES \\
\hline 1 & J.A.S. & 13 & $A T+T G A+E P$ & 6 meses & Regular & $88 \%-72 \%(18 \%)$ & $\cdot$ \\
\hline 3 & R.G. & 6 & $\begin{array}{l}\text { DVEVD+VS- } \\
\text { I+EP }\end{array}$ & 8 meses & Regular & $75 \%-71 \%(5 \%)$ & $\cdot$ \\
\hline 4 & A.C.P.T. & 13 & At.-Ib & 2 anos & Boa & $85 \%-52 \%(37 \%)$ & - \\
\hline 6 & G.P.S. & 13 & $A t+D S A-V+I M$ & 1 a 7 meses & Boa & $84 \%-54 \%(30 \%)$ & - \\
\hline 8 & L.A.B. & 7 & $A t-I b$ & 1 a 6 meses & Regular & $86 \%-43 \%(50 \%)$ & - \\
\hline 10 & S.G.P. & 7 & At-Ib & 1 a 5 meses & Baixa & $80 \%-70 \%(15 \%)$ & exaustão \\
\hline 11 & F.S.L. & 12 & AP c/ SIVI & 11 meses & Baixa & $85 \%-60 \%(20 \%)$ & - \\
\hline 14 & D.S.A. & 4 & $\begin{array}{l}\text { DVEVD+TGA+ } \\
\text { EP+Dex. }\end{array}$ & 5 meses & Baixa & $87 \%-86 \%(0,3 \%)$ & exaustão \\
\hline
\end{tabular}


MALUF, M. A.; ANDRADE, J. C. S.; CATANI, R.; CARVALHO, A. C.; NEGRINI, N.; BUFFOLO, E. - Avaliaçăo dos resultados tardios da operação de derivação cavo-pulmonar bidirecional, no tratamento paliativo de cardiopatias congênitas com câmara ventricular única. Rev. Bras. Cir. Cardiovasc., 9 (2): 95-103, 1994.

Uma adequada preservação miocárdica durante a operação é da maior relevância na evolução tardia destes pacientes onde apenas um ventrículo maneja toda a volemia do corpo.

A abordagem cirúrgica realizada mediante toracotomia mediana em todos os casos, consideramos muito confortável e segura, embora há relatos ${ }^{19}$ de abordagem por toracotomia direita.

Um grupo de pacientes de nossa série foi operado apenas com o uso de derivação VCS-AD, evitando-se assim o uso da CEC, da mesma forma que KOBAYASHI et alii ${ }^{19}$; nos casos em que foi usada a CEC, evitou-se o pinçamento da aorta e uso de solução cardioplégica, realizando-se o procedimento com o coração batando.

Não tivemos problemas com a feitura da anastomose, usando-se sutura contínua com fio reabsorvivel (PDS 6-0) em todos os casos. No coto proximal da VCS, foi suturado um selo de pericárdio bovino, para facilitar a anastomose com APD, no segundo tempo da operação.

Após realizar a anastomose cavo-pulmonar, foi interrompido o fluxo pulmonar através da sutura da valva pulmonar (VP) ou ligadura do B-T funcionante. Em 2 pacientes (casos de $n^{\circ} 6$ e $n^{\circ} 12$ ) o estudo ecodopplercardiográfico e cineangiográfico no $\mathrm{PO}$, detectaram fluxo sangüíneo através da VP, sem interferir no funcionamento da derivação cavo-pulmonar. KOBAYASHI et alii ${ }^{19}$, demonstraram os benefícios do fluxo pulmonar pulsátil, deixando aberta a VP estenótica ou B-T contralateral. Os autores relatam melhora do fluxo pulmonar, aumento da saturação arterial e diminuição da insuficiência da valva $A-V$, devido ao melhor esvaziamento ventricular.

Recentemente deixamos propositadamente aberta a VP estenótica de um paciente (caso de $\mathrm{n}$ 은 16), portador de AT tipo Ib, tendo boa evolução PO.
Houve $3(17.6 \%$ ) óbitos no POI devido a baixo débito cardíaco. As causas que levaram a má evolução talvez estejam relacionadas a inadequada complacência pulmonar ou dificuldade da drenagem da VCS, devido a importante circulação colateral para o pulmão.

$\mathrm{Na}$ nossa série tivemos apenas um paciente (caso de $n^{\circ}$ 12) que apresentou trombose venosa no POI. Optamos pela heparinização sistêmica e na ausência de melhora clínica indicamos a reintervenção para a retirada do trombo que comprometia a anastomose.

De rotina, não foi usado esquema de heparinização.

Doze $(70,5 \%)$ pacientes evoluíram bem após o primeiro tempo da operação. Um paciente (caso de $n^{\circ} 2$ ) apresentou queda progressiva da saturação e foi submetido ao segundo tempo da operação com intervalo de 22 meses.

Consideramos da maior importância o seguimento deste grupo de pacientes, coletando-se informações sobre fluxo pulmonar mediante a ecodopplercardiografia ou ressonância nuclear magnética e de perfusão pulmonar pela cintilografia radioisotópica. No entanto, os dados clínicos e a capacidade física, como resposta ao esforço, constituem os elementos primordiais para indicar o segundo tempo da operaçāo.

Três pacientes de nossa série apresentaram capacidade física baixa, com queda da saturação, sendo que 2 não conseguiram concluir o teste ergométrico, por exaustão.

Talvez, a avaliação dos dados obtidos e a confecção de um score permitiram determinar o momento ideal para a realização da operação definitiva. 
MALUF, M. A.; ANDRADE, J. C. S.; CATANI, R.; CARVALHO, A. C.; NEGRINI, N.; BUFFOLO, E. - Avaliação dos resultados tardios da operação de derivação cavo-pulmonar bidirecional, no tratamento paliativo de cardiopatias congênitas com câmara ventricular única. Rev. Bras. Cir. Cardiovasc., 9 (2): 95-103, 1994.

\section{RBCCV $44205-236$}

MALUF, M. A.; ANDRADE, J. C. S.; CATANI, R.; CARVALHO, A. C.: NEGRINI, N.; BUFFOLO, E. Assessment of late results of bidirecional cavopulmonary shunt on paliative treatment of congenital heart disease with functional isolated ventricular chamber. Rev. Bras. Cir. Cardiovasc., 9 (2): 95-103, 1993.

ABSTRACT: From March 1990 to January 1994, 17 patients 1 to 13 years old (mean 7 years), who had congenital heart disease with functional isolated ventricular chamber, were submitted to bidirectional cavopulmonary shunts. Nine cases had Tricuspid Atresia (TA) ( 7 type lb, 1 type Ic and 1 type Il b). Five cases had Double Inlet Right ventricle (DIRV); two cases had multiple Ventricular Septal Defects (VSD) plus RV hipoplasia. One case had pulmonary atresia with Intact Ventricular septum (PA/IVS). Nine $(52,9 \%$ ) patients had been submitted, to previous paliative operations. The operations in $12(70,5 \%)$ cases were with extracorporeal circulation (ECC) and in $5(29.4 \%$ ) cases with superior vena cava (SVC) to right atrio (RA) temporary shunt. In all cases the SVC was anastomosed to right pulmonary artery (RPA) closing the pulmonary valve (PV) or linking the BlalockTaussig shunt to interrupt the pulmonary flow. There are three $(17,6 \%)$ deaths in the immediate postoperative period and $2(14,2 \%)$ late deaths. Twelve $(70,5 \%)$ patients were followed-up for 2 to 46 months. One patient was submitted to the 2 nd operation, tunnelating the inferior vena cava (IVC) to RPA, with success. The assessment of bidirectional cavopulmonary flow was made with Doppler echocardiography and magnetic resonance and the pulmonary perfusion was made with radioisotopic cintilography. The indication for the $2 \mathrm{nd}$, operation depended on the clinical evolution and assessment of arterial saturation during the cicloergometric test. The bidirectional cavo pulmonary shunt allows the progressive adaptation of the venous flow to lung and reduced ventricular volume load, preparing the patient to total venous shunt.

DESCRIPTORS: cavopulmonary shunt, surgery.

\section{REFERÊNCIAS BIBLIOGRÁFICAS} 1 ABRAMS, L.D. - Side to side cavopulmonary
anastomosis for the palliation of "primitive ventricle". Br. Heart J., 39: 926, 1977. (Resumo).

CHOUSSAT, A.; FONTAN, F.; BESSE, P.; VALLOT, F.; CHAUVE, A.; BRICAUD, H. - Selection criteria for Tontam procedure. In: ANDERSON, R. H. \& SHINEBOURNE, W. A. (eds) Pediatric Cardiology 1977. Edinburgh, Scotland, Churchill Livingstone, Inc., 1978. p. 559 .

DE LEON, S. Y.; ILBAWI, M. N.; IDRISS, F. S.; MUSTER, A. J.; GIDDING, S. S.; BERRY, T. E.; PAUL, M. H. - Fontan type operation for complex lesions: surgical considerations to improve survival. J. Thorac. Cardiovasc. Surg., 92: 1029-1037, 1986.

DE LEON, S. Y.: ILBAWI, M. N.; IDRISS, F. S.; MUSTER, A. J.; GIDDING, S. S.; BERRY, T. E.; PAUL, M. H. - Persistent low cardiac output after the Fontan operation. Should takedown be considered. J. Thorac. Cardiovasc. Surg., 92: 402-405, 1986.

DE VIVIE, E. K. \& RUPPRATH, G. - Long-term results after Fontan procedure and its modifications. $J$ Thorac Cardiovasc. Surg., 91: 690-697, 1986 atresia. Thorax, 26: 240-248, 1971
7 GLENN, W. W. L. - Circulatory bypass of the right side of the heart. IV. Shunt between superior vena cava and distal right pulmonary artery: report of clinical application. N. Engl. J. Med., 259: 117-120, 1958.

8 HALLER, J. A.; ADKINS, J. C.; WORTHINGTON, M.; RAVENHORST, J. - Experimental studies on permanent bypass of the right heart. Surgery, 59: 1128-1132, 1966.

9 HOPKINS, R. A.; ARMSTRONG, B. E.; SERWER. G. A.: PETERSON. R. J.; OLDHAM, H. N. - Physiological rationale for a bidirectional cavopulmonary shunt: a versatile complement to the Fontan principle. J. Thorac. Cardiovasc. Surg., 90: 391-398, 1985.

10 HUMES, R. A.; FELDT, R. H.; PORTER, C. J.; JULSRUD, P. R.; PUGA, F. J.; DANIELSON, G. K. - The modified Fontan operation for asplenia and polysplenia syndromes. J. Thorac. Cardiovasc. Surg., 96: 212-218, 1988.

11 KAWASHIMA, Y.; KITAMURA, S.; MATSUDA, H.; SHIMAZAKI, Y.: NAKANO, S.: HIROSE, H. - Total cavopulmonary hunt operation in complex cardiac anomalies. J. Thorac. Cardiovasc. Surg., 87: 74$81,1984$.

12 KIRKLIN, J. K.; BLACKSTONE, H.; KIRKLIN, J. W.; PACIFICO, A. D.; BARGERON Jr., L. M. - The Fontan 
MALUF, M. A.; ANDRADE, J. C. S.; CATANI, R.; CARVALHO, A. C.; NEGRINI, N.; BUFFOLO, E. - Avaliação dos resultados tardios da operação de derivação cavo-pulmonar bidirecional, no tratamento paliativo de cardiopatias congênitas com câmara ventricular única. Rev. Bras. Cir. Cardiovasc., 9 (2): 95-103, 1994.

operation: ventricular hypertrophy, age, and date of operation as risk factors. J. Thorac. Cardiovasc. Surg., 92: 1049-1064,1986.

KOBAYASHI, J.; MATSUDA, H.; NAKANO, S.; SHIMAZAKI, Y.; IKAWA, S.; MITSUNO, M.; TAKAHASHI, Y.; KAWASHIMA, Y.; ARISAWA, J.; MATSUSHITA, T. Hemodynamics effects of bidirectional cavopulmonary shunt with pulsatile pulmonary flow. Circulation, 5 (Supl. 3): 219-225, 1984.

KREUTZER, G. O.: VARGAS, F. J.: SCHLICHTER, A. J.: LAURA, J. P.: SUAREZ, J. C.; CORONEL. A. R.; KREUTZER, E. A. - Atriopulmonary anastomosis. J. Thorac. Cardiovasc. Surg., 83: 427, 1982.

LAKS, H.; HAAS, G. S.; PEARL, J. M.; SADEGHI, A. M.; GEORGE, B.; SANTULLI, T. V.; WILLIAMS, R. G. The use of an adjustive intra-atrial communication in patients undergoing the Fontan and definitive heart procedures. Circulation, 78 (Supl. 3): II-357. 1988.

LAKS, H.; PEARL, J. M.; HAAS, G. S.; DRINKWATER,
D. C.; MILGALTER, E.; JARMAKANI, J. M.; ISABELJONES. J.; GEORGE, B. L.; WILLIAMS, R. G. Partial Fontan: advantage of an adjustable interatrial comunication. Ann. Thorac. Surg., 52: 1084-1085, 1991.

17 MAYER Jr., J. E.; HELGASON, H.; JONAS, R. A.; LANG, P.; VARGAS, F. J.; COOK. N.; CASTANEDA, A. R. - Extending the limits for modified Fontan procedures. J. Thorac. Cardiovasc. Surg., 92: 1021-1028, 1986.

18 MAZZERA, E.; CORNO, A.; PICARDO, S.; DI DONATO, R.; MARINO, B.; COSTA, D.: MARCELLETTI, C. Bidirectional cavopulmonary shunts: clinical applications as staged or definitive palliation. Ann. Thorac. Surg., 47: 415-420, 1989.

19 STELLIN, G.; MAZZUCCO, A.; BORTOLOTTI, U.; DER TORSO, S.; FAGGIAN, G.; FRACASSO, A.; LIVI, U.; MILANO, U.; RIZZOLI, G.; GALLUCI, V. Tricuspid atresia versus other complex lesions: comparison of results with a modified Fontan procedure. J. Thorac. Cardiovasc. Surg., 96: 204211, 1988. 